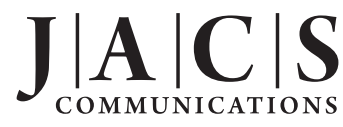

Published on Web 04/20/2010

\title{
Controllable Growth and Field-Effect Property of Monolayer to Multilayer Microstripes of an Organic Semiconductor
}

\author{
Liqiang Li, ${ }^{\dagger}$ Peng Gao, ${ }^{\ddagger}$ Klaus C. Schuermann, ${ }^{\dagger}$ Stefan Ostendorp,${ }^{\dagger}$ Wenchong Wang, ${ }^{\dagger}$ Chuan Du, ${ }^{\dagger}$ \\ Yong Lei, ${ }^{\dagger}$ Harald Fuchs, ${ }^{\dagger}$ Luisa De Cola,${ }^{\dagger}$ Klaus Müllen, ${ }^{*, \neq}$ and Lifeng Chi ${ }^{\star, \dagger}$ \\ Physikalisches Institut and Center for Nanotechnology (CeNTech), Universität Münster, 48149 Münster, Germany, \\ and Max Planck Institute for Polymer Research, Ackermannweg 10, 55128 Mainz, Germany
}

Received March 1, 2010; E-mail: chi@uni-muenster.de; muellen@mpip-mainz.mpg.de

The electronic properties of organic semiconductors are mainly governed by both their molecular structure and the molecular packing state which is significantly influenced by the growth process. ${ }^{1-3}$ Therefore, controlled growth of organic semiconductors at a molecular level such as in precise layer thickness (from monolayer to multilayers), molecular ordering, and orientation is an important but challenging topic for fundamental research and practical application. ${ }^{2-4}$ Here we show controllable growth of partially aligned monolayer to multilayer micrometer stripes over large areas via a dip-coating method. Based on this unique growth, we further investigate the field-effect property of these microstripes with different numbers of molecular layers.

In organic field-effect transistors (OFETs) and OFETs-based sensors, the first few layers of organic semiconductors, where charge accumulation and transporting mainly occur, will determine the device performance. ${ }^{3}$ However, OFETs based on ultrathin films are rarely reported because of the difficulty to grow large-area, high quality, and continuous ultrathin films. Until now, growth of ultrathin organic semiconductor films with monolayer precision is generally obtained by vacuum deposition, ${ }^{4}$ LB techniques,,${ }^{5 a}$ and electrostatic force-based self-assembly. ${ }^{5 \mathrm{~b}}$ However, the first method usually yields discontinuous patches because of 3D island growth. The LB technique and the electrostatic force-based self-assembly are restricted to compounds with special functional groups which may compromise structural ordering and device performance. More importantly, it is difficult, if not impossible, for all these methods to realize aligned 1D organic semiconductor structure with a controllable number of molecular layers.

Our approach to grow aligned microstripes on silicon with monolayer precision is based on dip-coating (Figure 1b) with varying pulling speed. A tetrathieno analogue of pentacene (DTBDT-C9, Figure 1a) was employed in this work as a prototype case. The selection of the compound is dependent on (1) the strong $\pi-\pi$ interaction between the large aromatic plane and van der Waals interactions between long alkyl chains which promote the self-assembly ability to form ordered and regular nano/microstructures and (2) the ability of the series of compounds to exhibit excellent field-effect properties. ${ }^{6}$

During the dip-coating process, the pulling speed $(U)$ was systematically adjusted and appeared to have a great influence on the growth of DTBDT-C9, mainly on the number of molecular layers $(N)$ of microstripes, as well as on their fractal morphology. Remarkably enough, by such a simple pulling method, monolayer and bilayer stripes could be created over large areas, as revealed by atomic force microscopy (AFM) images (Figure 1c and 1d; for stripes with variable layer numbers see Figure S1). The surface of

\footnotetext{
†Universität Münster.
}

* Max Planck Institute for Polymer Research.

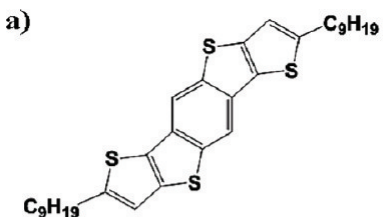

b)
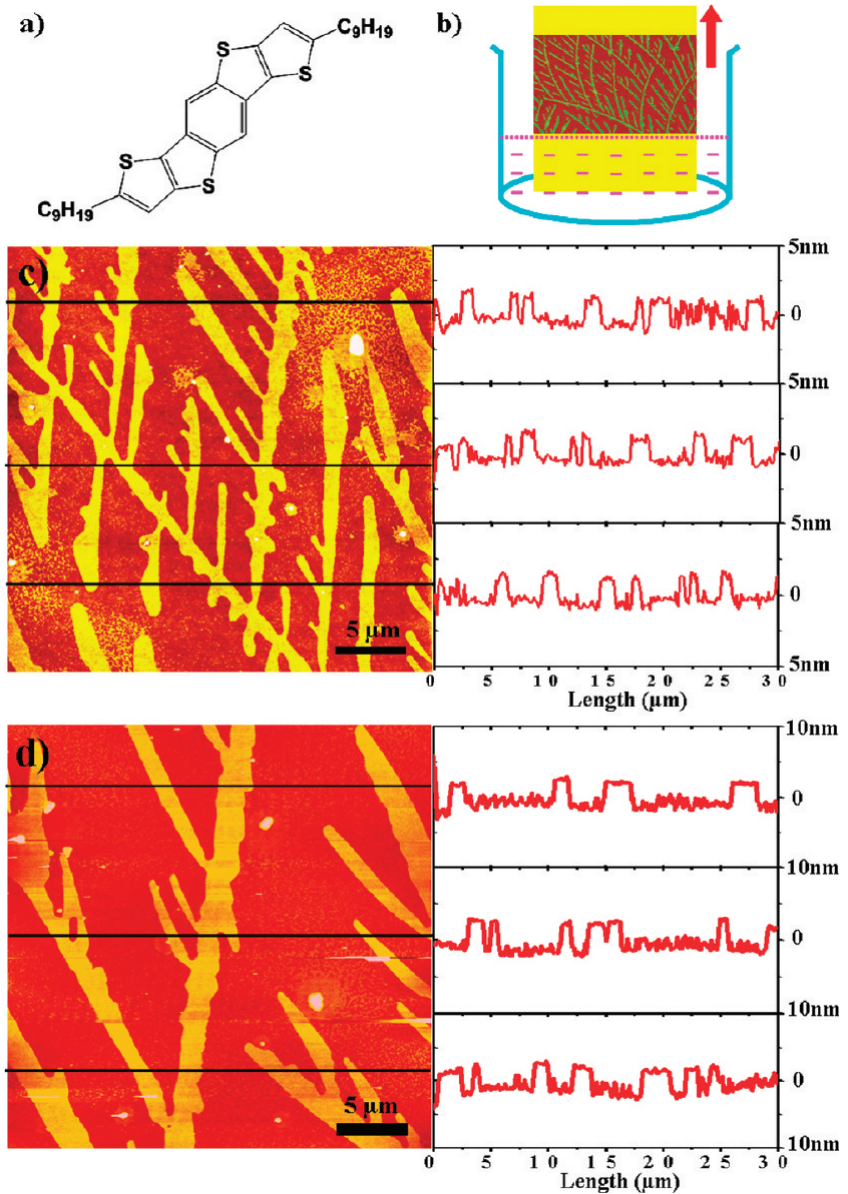

Figure 1. (a) Molecular structure of DTBDT-C9. (b) Schematic diagram of dip-coating process. AFM image and section analysis of (c) monolayer and (d) bilayer microstripes.

the stripes is smooth (rms, 3-5 $\AA$ ). The height along the stripes with lengths from tens to hundreds of micrometers is uniform, and the height of a monolayer $(d 1)$ is $\sim 2.06 \mathrm{~nm}$. The corresponding value of multilayers $(d \mathrm{n})$ fits the equation $d \mathrm{n}=N^{*} d 1$, where $N$ is the number of monolayers.

Figure 2 describes the relationship between the number of molecular layers $(N)$ and the pulling speed $(U)$. The number and the distribution of the molecular layers decrease with increasing speed; i.e., a higher pulling speed results in thinner and more uniform layers. At a pulling speed below $20 \mu \mathrm{m} / \mathrm{s}$, the stripes with three to nine monolayers always occur together. The optical image of large-area stripes formed at $5 \mu \mathrm{m} / \mathrm{s}$ reveals a dendritic structure (Figure 3a). At a pulling speed of $30 \mu \mathrm{m} / \mathrm{s}$, bilayer and monolayer microstripes were formed (for their optical images, see Figure $3 b$ 


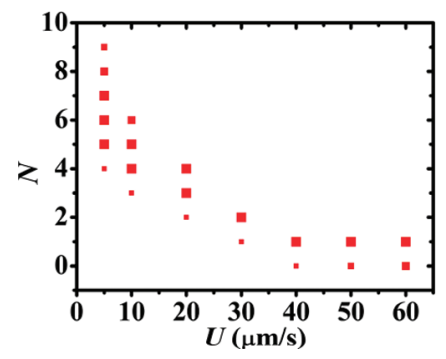

Figure 2. Evolution of the number of molecular layers $(N)$ with pulling speed $(U)$. The size of the symbol represents the schematic (not real) distribution of each layer at a given speed. 0 layer denotes a discontinuous island.

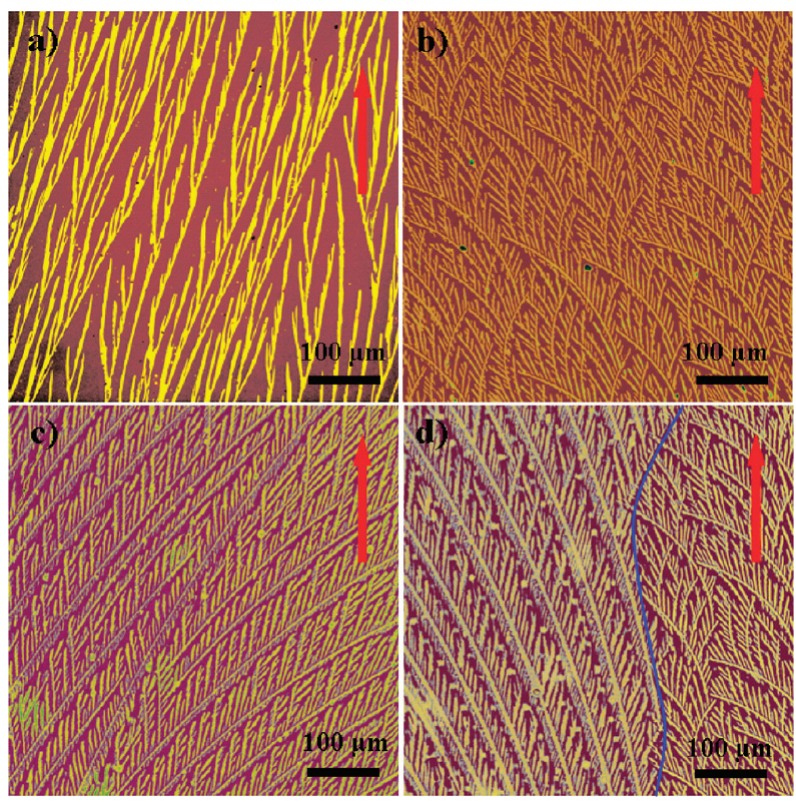

Figure 3. Optical images of large-area stripes of (a) mixed multilayer, (b) pure bilayer, (c) pure monolayer, and (d) cross area between bilayers (right part) and monolayers (left part). The red arrows indicate the pulling direction. The blue curve in d is marked as boundary line between bilayers and monolayers for better inspection.

and 3c). More interestingly, the bilayer and monolayer stripes are well separated from each other (Figure 3d) and are thus located at different areas of the substrate. Large area $\left(30 \times 30 \mu \mathrm{m}^{2}\right)$ AFM images of monolayer and bilayer stripes (as shown in Figure 1c,d) as well as AFM measurements at different locations guided by the microscope convincingly verify their uniformity. AFM measurements also reveal that the trunk of the dendritic monolayer and bilayer stripes is continuous and grain-free over tens to hundreds of micrometers. The continuity and length of individual stripes as well as the uniform large-area coverage facilitate the fabrication of electronic devices. At $40 \mu \mathrm{m} / \mathrm{s}$, a majority of large area monolayer stripes and a minority of discontinuous islands can be obtained. The morphology of monolayer stripes at this speed is similar to the monolayer region formed at $30 \mu \mathrm{m} / \mathrm{s}$ (Figure 3c). Further accelerating the speed to $60 \mu \mathrm{m} / \mathrm{s}$ leads to monolayer stripes and more discontinuous islands (Figure S1a).

Figure 3 reveals two further important characteristics. The first one is the dependence of stripe morphology on the pulling speed or the number of molecular layers. In principle, pulling the substrate from DTBDT-C9 solution yields a dendritic or fractal structure, but the fine structure is different; i.e., the morphology and the coverage of stripes vary with the number of molecular layers. This phenomenon must be due to different growth dynamics and/or fluid
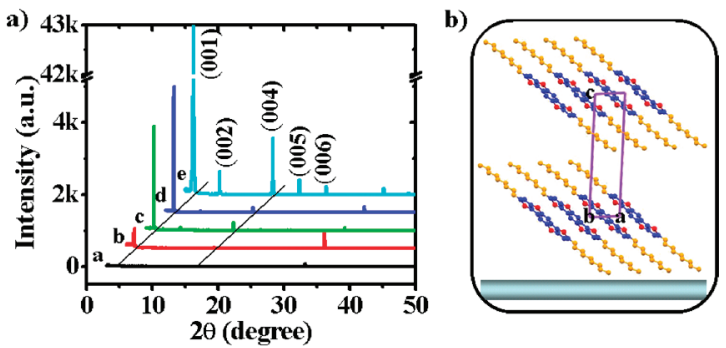

Figure 4. (a) XRD patterns of stripes with different layers. Curve a, monolayer; b, bilayer; c, 3-5 monolayer; d, 4-9 monolayer; e, thick film prepared by drop-casting. Curves $b-e$ are shifted horizontally with a step of $3^{\circ}$. (b) Schematic diagram of DTBDT molecule packing on the substrate.

flow field in the meniscus near the contact line at different pulling speeds. This feature of layer number-dependent morphology allows one to easily determine the number of monolayers in the stripes. The second finding is that all the stripes at different pulling speeds are partially aligned along the pulling direction. Proper alignment of one-dimensional structures will ensure optimal and reproducible electronic properties as needed in device fabrication.

The self-assembly phenomenon reported here, i.e., control over morphology, alignment, and the number of molecular layers arises from the growth mechanism of DTBDT-C9 molecules in the dipcoating process. The self-assembly of organic semiconductors in dip-coating is essentially an evaporation-controlled process that involves competing influences at the contact line among the evaporation rate of solvent, pulling speed of the substrate, carrying speed of solute by capillary force, and self-assembly speed of solute molecules. ${ }^{7}$ Systematic studies about the influence of experimental parameters including solvent, environment, concentration, temperature, and substrate on growth processes is ongoing. Theoretical modeling will be helpful to understand these complex kinetic processes $^{8}$ which is in progress as well.

To characterize the structure of these stripes, XRD measurements with the $\theta-2 \theta$ scan mode were performed (Figure 4a). Multilayer $(N \geq 2)$ stripes yield multiple peaks at $n \times 4.2^{\circ}(n \geq 1)$, which are attributed to the diffraction of a (001) crystalline lattice. This result is indicative of high molecular ordering within the multilayer microstripes. The exclusive appearance of a (001) peak for multilayer $(N \geq 2)$ stripes indicates that the (001) plane of the DTBDT-C9 crystalline is parallel to the substrate; i.e., the DTBDTC9 molecule obliquely stands on the substrates (edge-on molecular orientation, as shown in Figure $4 \mathrm{~b}$ ). The angle between the molecular plane and surface normal is calculated to be $43.89^{\circ}$. This type of edge-on orientation is beneficial to obtain good field-effect properties because the $\pi-\pi$ stacking plane is consistent with the charge transporting direction. ${ }^{9}$ From the XRD pattern, the height of the monolayer can be calculated as $\sim 2.10 \mathrm{~nm}$ which is nearly equal to the value $(2.06 \mathrm{~nm})$ measured by AFM.

The growth of aligned stripes with a controllable number of monolayers provides an elegant and practical experimental system to study the charge transporting property in the real charge accumulation and transporting layer of OFETs. Therefore, topcontact transistors with a channel length of $20 \mu \mathrm{m}$ were fabricated on these microstripes, especially on pure monolayer and bilayer microstripes. The continuity of the stripes eliminates the influence of grain boundaries on the field effect and discloses the intrinsic electrical transporting characteristics of the semiconductor material. Interestingly, the FET measurements indicate that multilayer $(N \geq$ 2) stripes exhibit good field-effect properties, while almost no fieldeffect characteristics can be detected for the monolayer stripes, both 

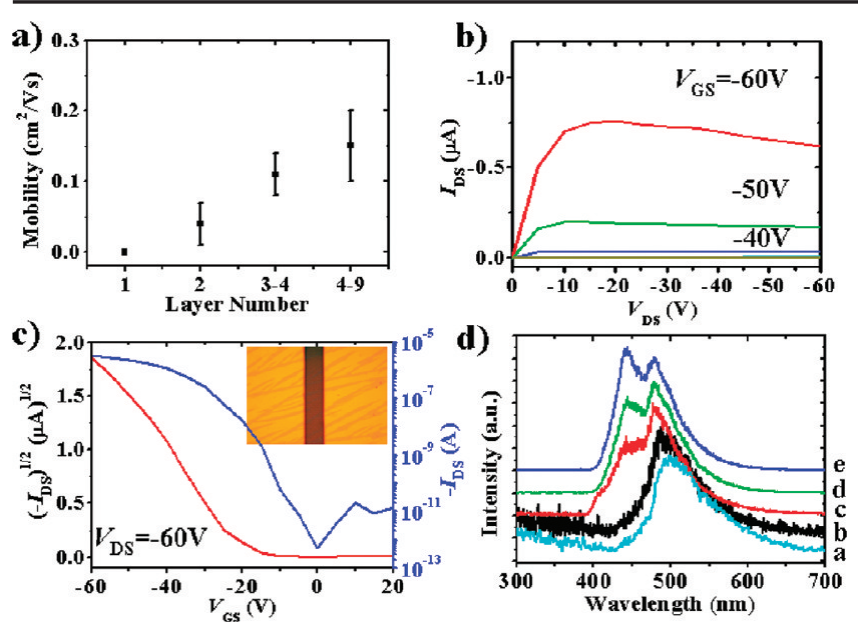

Figure 5. (a) Relationship between mobility and layer number of stripes. (b) Output and (c) transfer curves of OFETs with bilayer stripes. Inset in $\mathrm{c}$ is the optical image of OFETs device; the channel length is $20 \mu \mathrm{m}$. (d) Confocal fluorescence spectra of (a) amorphous film, (b) monolayer stripe, (c) bilayer stripe, (d) multilayer stripe, and (e) single crystal.

in air and under vacuum. Figure 5a shows the evolution of fieldeffect mobility with the number of molecular layers. It can be seen that the mobility increases, but not significantly, with the number of molecular layers. Furthermore, the threshold voltage for all the different stripes is in the range -20 to $-40 \mathrm{~V}$, disclosing no obvious dependence on the number of molecular layers. Figure $5 b$ and $c$ display the output and transfer curves of the device of bilayer stripes, while the inset in Figure 5c shows the optical image of the device on multiple bilayer stripes. These data provide firm evidence that the bilayer stripes possess well-behaved field-effect characteristics. From the transfer curves (Figure $5 \mathrm{c}$ ), the mobility $(\mu)$ of bilayer devices in the saturation region, threshold voltage $\left(V_{\mathrm{T}}\right)$, and on/off ratio are determined to be $0.06 \mathrm{~cm}^{2} \mathrm{~V}^{-1} \mathrm{~s}^{-1},-20.5 \mathrm{~V}$, and $8.9 \times$ $10^{5}$, respectively. The maximum mobility $\left(0.19 \mathrm{~cm}^{2} \mathrm{~V}^{-1} \mathrm{~s}^{-1}\right)$ of $3-9$ molecular layers is comparable to that $\left(0.1-0.2 \mathrm{~cm}^{2} \mathrm{~V}^{-1} \mathrm{~s}^{-1}\right)$ of thick films prepared by drop-casting (the electrical curves of the devices on multilayers $(N \geq 3)$ are given in Figure S2).

To understand why monolayer stripes fail to reveal a field effect, confocal fluorescence microscopy was applied for further characterization in view of the spectral and spatial resolution. As clearly seen in Figure 5d, the fluorescence spectra of monolayer (curve b) and multilayer ( $N \geq 2$, curves $\mathrm{c}$ and d) are different: the former one is similar to that of an amorphous film (curve a, deposited at the substrate temperature of approximately $-100{ }^{\circ} \mathrm{C}$ from the gas phase, showing a mobility lower than $10^{-6} \mathrm{~cm}^{2} \mathrm{~V}^{-1} \mathrm{~s}^{-1}$ ), while curves $\mathrm{c}$ and $\mathrm{d}$ are similar to that of a single crystal (curve e, prepared by slow evaporation of solvent from a diluted toluene solution). This result strongly suggests that a monolayer stripe is amorphous, while multilayer stripes consist of crystalline states, which is also confirmed by XRD measurements. The peak at 440 $\mathrm{nm}$ for multilayer $(N \geq 2)$ stripes is somewhat lower than that of the single crystal, which may be due to the lower crystallization degree or the presence of amorphous aggregates. The amorphous character of a monolayer stripe is most probably induced by the mismatch between the crystalline lattice of DTBDT and the substrate and/or short self-assembly time due to quick solvent evaporation at faster pulling speeds. Consequently, the presence of an amorphous state accounts for the lack of field effects in monolayer stripes.

In summary, this work presents the controllable growth of aligned monolayer to multilayer microstripes of a prototype organic semiconductor (DTBDT-C9) by varying the pulling speed in a dipcoating process. The number of molecular layers decreases with increased pulling speed. Lower pulling speeds yield mixed multilayers (3-9 monolayers). It is noteworthy that pure monolayer and bilayer microstripes over large areas can be obtained at high pulling speeds. The morphology of stripes exhibits a strong dependence on pulling speed as well. Based on this unique growth, FET devices were fabricated on these stripes. No field effect could be detected in monolayer stripes due to its amorphous character, while good field-effect behavior was found in multilayer $(N \geq 2)$ stripes. The controllable growth from monolayers to multilayers offers a powerful experimental system for fundamental research into real charge accumulation and transporting layers for OFETs.

Acknowledgment. This work was financially supported by the DFG-NSFC Transregio Project (TRR61).

Supporting Information Available: Experimental method, AFM images of stripe with different layers, electrical curves of devices on multilayer stripes. This material is available free of charge via the Internet at http://pubs.acs.org.

\section{References}

(1) (a) Forrest, S. R. Nature 2004, 428, 911-918. (b) Dimitrakopoulos, C. D.; Malenfant, P. R. L. Adv Mater 2002, 14, 99-117. (c) Katz, H. E.; Bao, Z. N.; Gilat, S. L. Acc. Chem. Res. 2001, 34, 359-369. (d) Mushrush, M.; Facchetti, A.; Lefenfeld, M.; Katz, H. E.; Marks, T. J. J. Am. Chem. Soc. 2003, 125, 9414-9423.

(2) (a) Loi, M. A.; Da Como, E.; Dinelli, F.; Murgia, M.; Zamboni, R.; Biscarini, F.; Muccini, M. Nat. Mater. 2005, 4, 81-85. (b) Hong, Y. N.; Lam, J. W. Y.; Tang, B. Z. Chem. Commun. 2009, 4332-4353. (c) Dimitrakopoulos, C. D.; Brown, A. R.; Pomp, A. J. Appl. Phys. 1996, 80, 2501-2508.

(3) (a) Dinelli, F.; Murgia, M.; Levy, P.; Cavallini, M.; Biscarini, F.; de Leeuw, D. M. Phys. Rev. Lett. 2004, 92, 116802. (b) Huang, J.; Sun, J.; Katz, H. E. Adv. Mater. 2008, 20, 2567-2572.

(4) (a) Sirringhaus, H.; Brown, P. J.; Friend, R. H.; Nielsen, M. M.; Bechgaard, K.; Langeveld-Voss, B. M. W.; Spiering, A. J. H.; Janssen, R. A. J.; Meijer, E. W.; Herwig, P.; de Leeuw, D. M. Nature 1999, 401, 685-688. (b) Briseno, A. L.; Mannsfeld, S. C. B.; Ling, M. M.; Liu, S. H.; Tseng, R. J.; Reese, C.; Roberts, M. E.; Yang, Y.; Wudl, F.; Bao, Z. N. Nature 2006, 444, 913-917. (c) Li, L.; Tang, Q.; Li, H.; Yang, X.; Hu, W.; Song, Y.; Shuai, Z.; Xu, W.; Liu, Y.; Zhu, D. Adv. Mater. 2007, 19, 2613-2617. (d) Kline, R. J.; McGehee, M. D.; Toney, M. F. Nat. Mater. 2006, 5, 222-228. Zang, L.; Che, Y. K.; Moore, J. S. Acc. Chem. Res. 2008, 41, 1596-1608. (e) Liu, S. H.; Wang, W. C. M.; Briseno, A. L.; Mannsfeld, S. C. E.; Bao, Z. N. Adv. Mater. 2009, 21, 1217-1232.

(5) (a) Xiao, K.; Liu, Y. Q.; Huang, X. B.; Xu, Y.; Yu, G.; Zhu, D. B. J. Phys. Chem. B 2003, 107, 9226-9230. (b) Locklin, J.; Shinbo, K.; Onishi, K.; Kaneko, F.; Bao, Z. N.; Advincula, R. C. Chem. Mater. 2003, 15, 14041412.

(6) Gao, P.; Beckmann, D.; Tsao, H. N.; Feng, X. L.; Enkelmann, V.; Baumgarten, M.; Pisula, W.; Müllen, K. Adv. Mater. 2009, 21, 213-216.

(7) (a) Liu, N.; Zhou, Y.; Wang, L.; Peng, J.; Wang, J.; Pei, J.; Cao, Y. Langmuir 2008, 25, 665-671. (b) Huang, J. X.; Fan, R.; Connor, S.; Yang, P. D. Angew. Chem., Int. Ed. 2007, 46, 2414-2417.

(8) Thiele, U.; Vancea, I.; Archer, A. J.; Robbins, M. J.; Frastia, L.; Stannard, A.; Pauliac-Vaujour, E.; Martin, C. P.; Blunt, M. O.; Moriarty, P. J. J. Phys.: Condens. Matter 2009, 21, 264016.

(9) (a) Wu, Y.; Li, Y.; Gardner, S.; Ong, B. S. J. Am. Chem. Soc. 2004, 127, 614-618. (b) Merlo, J. A.; Newman, C. R.; Gerlach, C. P.; Kelley, T. W.; Muyres, D. V.; Fritz, S. E.; Toney, M. F.; Frisbie, C. D. J. Am. Chem. Soc. 2005, 127, 3997-4009.

JA1017267 